\title{
MINIMUM ENERGY CONTROL OF FRACTIONAL POSITIVE CONTINUOUS-TIME LINEAR SYSTEMS WITH BOUNDED INPUTS
}

\author{
TADEUSZ KACZOREK \\ Faculty of Electrical Engineering \\ Białystok University of Technology, ul. Wiejska 45D, 15-351 Białystok, Poland \\ e-mail:kaczorek@isep.pw.edu.pl
}

\begin{abstract}
A minimum energy control problem for fractional positive continuous-time linear systems with bounded inputs is formulated and solved. Sufficient conditions for the existence of a solution to the problem are established. A procedure for solving the problem is proposed and illustrated with a numerical example.
\end{abstract}

Keywords: fractional systems, positive systems, minimum energy control, bounded inputs.

\section{Introduction}

Mathematical fundamentals of fractional calculus are given in the monographs of Oldham and Spanier (1974), Ostalczyk (2008) as well as Podlubny (1999). An overview of the state of the art in fractional systems theory is presented by Kaczorek (1992). Some recent interesting results in fractional systems theory and its applications can be found in the works of Dzieliński et al. (2009), Kaczorek (2011a; 2008c), Radwan et al. (2009), Solteiro Pires et al. (2006) or Vinagre (2002).

A dynamical system is called positive if its trajectory starting from any nonnegative initial state remains forever in the positive orthant for all nonnegative inputs (Farina and Rinaldi, 2000; Kaczorek, 2001). A variety of models having positive behavior can be found in engineering, economics, social sciences, biology and medicine, etc.

Positive fractional linear systems were investigated by Kaczorek (2008a; 2011b; 2011c; 2012), while the stability of fractional linear 1D discrete-time and continuous-time systems was discussed by Busłowicz (2008), Dzieliński and Sierociuk (2008) as well as Kaczorek (2012), who also studied the stability of 2D fractional positive linear systems (Kaczorek, 2009). The notion of practical stability of positive fractional discrete-time linear systems was introduced by Kaczorek (2008b), who also addressed the positivity of descriptor linear systems by the use of the shuffle algorithm (Kaczorek, 2011d). The minimum energy control problem for standard linear systems was formulated and solved by Klamka (1991; 1983; 1976a), and for 2D linear systems with variable coefficients by Kaczorek and Klamka (1986). The controllability and minimum energy control problem of fractional discrete-time linear systems was investigated by Klamka (2010), while minimum energy control of fractional positive continuous-time linear systems was addressed by Kaczorek (2013b; 2013c; 2014; 1992), along with its counterpart for descriptor positive discrete-time linear systems (Kaczorek, 2014a; 2013c; 2014b;1992). Minimum energy control of positive continuous-time systems with bounded inputs was studied by the same author (Kaczorek, 2013d).

In this paper the minimum energy control problem for fractional positive continuous-time linear systems with bounded inputs will be formulated and solved. The paper is organized as follows. In Section 2, basic definitions and theorems of fractional positive continuous-time linear systems are recalled, and the necessary and sufficient conditions for the reachability of the systems are given. The main result of the paper is given in Section 3, where the minimum energy control problem is formulated and sufficient conditions for its solution are established. A procedure and an illustrating numerical example are presented in Section 4. Concluding remarks are given in Section 5 .

The following notation will be used: $\mathbb{R}$, the set of real numbers; $\mathbb{R}^{n \times m}$, the set of $n \times m$ real matrices; $\mathbb{R}_{+}^{n \times m}$, the set of $n \times m$ matrices with nonnegative entries and $\mathbb{R}_{+}^{n}=\mathbb{R}_{+}^{n \times 1} ; M_{n}$, the set of $n \times n$ Metzler matrices (real 
matrices with nonnegative off-diagonal entries); $I_{n}$, the $n \times n$ identity matrix.

\section{Preliminaries}

The following Caputo definition of the fractional derivative will be used (Kaczorek, 2012; Oldham and Spanier, 1974; Ostalczyk, 2008; Podlubny, 1999):

$$
\begin{array}{r}
\mathrm{D}^{\alpha} f(t) \\
=\frac{\mathrm{d}^{\alpha}}{\mathrm{d} t^{\alpha}} f(t)=\frac{1}{\Gamma(n-\alpha)} \int_{0}^{t} \frac{f^{(n)}}{(t-\tau)^{\alpha+1-n}} \mathrm{~d} \tau, \\
n-1<\alpha \leq n \in \mathbb{N}=\{1,2, \ldots\},
\end{array}
$$

where $\alpha \in \mathbb{R}$ is the order of fractional derivative and

$$
f^{(n)}(\tau)=\frac{\mathrm{d}^{n} f(\tau)}{\mathrm{d} \tau^{n}},
$$

while

$$
\Gamma(x)=\int_{0}^{\infty} e^{-t} t^{x-1} \mathrm{~d} t
$$

is the gamma function.

Consider a continuous-time fractional linear system described by the state equation

$$
\mathrm{D}^{\alpha} x(t)=A x(t)+B u(t), \quad 0<\alpha \leq 1,
$$

where $x(t) \in \mathbb{R}^{n}, u(t) \in \mathbb{R}^{m}$ are the state and input vectors and $A \in \mathbb{R}^{n \times n}, B \in \mathbb{R}^{n \times m}$.

Theorem 1. The solution of Eqn. (2) is given by

$$
x(t)=\Phi_{0}(t) x_{0}+\int_{0}^{t} \Phi(t-\tau) B u(\tau) \mathrm{d} \tau, \quad x(0)=x_{0},
$$

where

$$
\begin{gathered}
\Phi_{0}(t)=E_{\alpha}\left(A t^{\alpha}\right)=\sum_{k=0}^{\infty} \frac{A^{k} t^{k \alpha}}{\Gamma(k \alpha+1)}, \\
\Phi(t)=\sum_{k=0}^{\infty} \frac{A^{k} t^{(k+1) \alpha-1}}{\Gamma[(k+1) \alpha]}
\end{gathered}
$$

and $E_{\alpha}\left(A t^{\alpha}\right)$ is the Mittage-Leffler matrix function (Kaczorek, 2012).

Definition 1. (Kaczorek, 2012) The fractional system (2) is called (internally) positive fractional if and only if $x(t) \in \mathbb{R}_{+}^{n}$ for $t \geq 0$ for any initial conditions $x_{0} \in \mathbb{R}_{+}^{n}$ and all inputs $u(t) \in \mathbb{R}_{+}^{m}, t \geq 0$.

Theorem 2. (Kaczorek, 2012) The continuous-time fractional system (2) is (internally) positive if and only if the matrix $A$ is a Metzler matrix and

$$
A \in M_{n}, \quad B \in \mathbb{R}_{+}^{n \times m} .
$$

Definition 2. The state $x_{f} \in \mathbb{R}_{+}^{n}$ of the fractional system (2) is called reachable in time $t_{f}$ if there exists an input $u(t) \in \mathbb{R}_{+}^{m}, \quad t \in\left[0, t_{f}\right]$, which steers the state of the system (2) from zero initial state $x_{0}=0$ to the state $x_{f}$.

A real square matrix is called monomial if each its row and each its column contains only one positive entry and the remaining entries are zero.

Theorem 3. The positive fractional system (2) is reachable in time $t \in\left[0, t_{f}\right]$ if and only if the matrix $A \in M_{n}$ is diagonal and the matrix $B \in \mathbb{R}_{+}^{n \times n}$ is monomial.

Proof. (Sufficiency) It is well known (Kaczorek, 2001; 2012) that if $A \in M_{n}$ is diagonal then $\Phi(t) \in \mathbb{R}_{+}^{n \times n}$ is also diagonal, and if $B \in \mathbb{R}_{+}^{n \times m}$ is monomial then $B B^{T} \in \mathbb{R}_{+}^{n \times n}$ is also monomial. In this case the matrix

$$
R_{f}=\int_{0}^{t_{f}} \Phi(\tau) B B^{T} \Phi^{T}(\tau) \mathrm{d} \tau \in \mathbb{R}_{+}^{n \times n}
$$

is also monomial and $R_{f}^{-1} \in \mathbb{R}_{+}^{n \times n}$. The input

$$
u(t)=B^{T} \Phi^{T}\left(t_{f}-t\right) R_{f}^{-1} x_{f}
$$

steers the state of the system (2) from $x_{0}=0$ to $x_{f}$ since, using (3) for $x_{0}=0$ and (5), we obtain

$$
\begin{aligned}
x\left(t_{f}\right) & =\int_{0}^{t_{f}} \Phi\left(t_{f}-\tau\right) B u(\tau) \mathrm{d} \tau \\
& =\int_{0}^{t_{f}} \Phi\left(t_{f}-\tau\right) B B^{T} \Phi^{T}\left(t_{f}-\tau\right) \mathrm{d} \tau R_{f}^{-1} x_{f} \\
& =\int_{0}^{t_{f}} \Phi(\tau) B B^{T} \Phi^{T}(\tau) \mathrm{d} \tau R_{f}^{-1} x_{f}=x_{f} .
\end{aligned}
$$

The proof of necessity is given by Kaczorek (2013a).

\section{Problem formulation and its solution}

Consider the fractional positive system (2) with diagonal $A \in M_{n}$ and monomial $B \in \mathbb{R}_{+}^{n \times m}$. If the system is reachable in time $t \in\left[0, t_{f}\right]$, then usually there exist many different inputs $u(t) \in \mathbb{R}_{+}^{n}$ that steer the state of the system from $x_{0}=0$ to $x_{f} \in \mathbb{R}_{+}^{n}$. Among these inputs we are looking for an input $u(t) \in \mathbb{R}_{+}^{n}, t \in\left[0, t_{f}\right]$ satisfying the condition

$$
u(t) \leq U \in \mathbb{R}_{+}^{n}, \quad t \in\left[0, t_{f}\right]
$$

that minimizes the performance index

$$
I(u)=\int_{0}^{t_{f}} u^{T}(\tau) Q u(\tau) \mathrm{d} \tau
$$

where $Q \in \mathbb{R}_{+}^{n \times n}$ is a symmetric positive-definite matrix and $Q^{-1} \in \mathbb{R}_{+}^{n \times n}$. 
The minimum energy control problem for the fractional positive continuous-time linear system (2) can be stated as follows: Given matrices $A \in M_{n}, B \in$ $\mathbb{R}_{+}^{n \times m}, \alpha, U \in \mathbb{R}_{+}^{n}$ and $Q \in \mathbb{R}_{+}^{n \times n}$ of the performance index 11$], x_{f} \in \mathbb{R}_{+}^{n}$ and $t_{f}>0$, find an input $u(t) \in \mathbb{R}_{+}^{n}$ for $t \in\left[0, t_{f}\right]$ satisfying (10) that steers the state vector of the system from $x_{0}=0$ to $x_{f} \in \mathbb{R}_{+}^{n}$ and minimizes the performance index (11).

To solve the problem, we define the matrix

$$
\begin{aligned}
& W\left(t_{f}\right) \\
& \quad=\int_{0}^{t_{f}} \Phi\left(t_{f}-\tau\right) B Q^{-1} B^{T} \Phi^{T}\left(t_{f}-\tau\right) \mathrm{d} \tau,
\end{aligned}
$$

where $\Phi(t)$ is given by (5). From (12) and Theorem 2 it follows that the matrix (12) is monomial if and only if the fractional positive system (2) is reachable in time $\left[0, t_{f}\right]$. In this case, we may define the input

$$
\hat{u}(t)=Q^{-1} B^{T} \Phi^{T}\left(t_{f}-t\right) W^{-1}\left(t_{f}\right) x_{f}
$$

for $t \in\left[0, t_{f}\right]$.

Note that the input (13) satisfies the condition $u(t) \in$ $\mathbb{R}_{+}^{n}$ for $t \in\left[0, t_{f}\right]$ if

$$
Q^{-1} \in \mathbb{R}_{+}^{n \times n}
$$

and

$$
W^{-1}\left(t_{f}\right) \in \mathbb{R}_{+}^{n \times n}
$$

Theorem 4. Let $\bar{u}(t) \in \mathbb{R}_{+}^{n}$ for $t \in\left[0, t_{f}\right]$ be an input satisfying (10) that steers the state of the fractional positive system (2) from $x_{0}=0$ to $x_{f} \in \mathbb{R}_{+}^{n}$. Then the input (13) satisfying (10) also steers the state of the system from $x_{0}=0$ to $x_{f} \in \mathbb{R}_{+}^{n}$ and minimizes the performance index (11), i.e.,

$$
I(\hat{u}) \leq I(\bar{u}) .
$$

The minimal value of the performance index (11) is equal to

$$
I(\hat{u})=x_{f}^{T} W^{-1}\left(t_{f}\right) x_{f} .
$$

Proof. If the conditions 14a are met, then the input (13) is well defined and $\hat{u}(t) \in \mathbb{R}_{+}^{n}$ for $t \in\left[0, t_{f}\right]$. We shall show that the input steers the state of the system from $x_{0}=0$ to $x_{f} \in \mathbb{R}_{+}^{n}$. Substitution of (13) into (3) for $t=t_{f}$ and $x_{0}=0$ yields

$$
x\left(t_{f}\right)=\int_{0}^{t_{f}} \Phi\left(t_{f}-\tau\right) B \hat{u}(\tau) \mathrm{d} \tau
$$

$$
\begin{aligned}
& =\int_{0}^{t_{f}} \Phi\left(t_{f}-\tau\right) B Q^{-1} B^{T} \Phi^{T}\left(t_{f}-\tau\right) \mathrm{d} \tau W_{f}^{-1} x_{f} \\
& =x_{f}
\end{aligned}
$$

since (12) holds. By assumption, the inputs $\bar{u}(t)$ and $\hat{u}(t)$, $t \in\left[0, t_{f}\right]$, steer the state of the system from $x_{0}=0$ to $x_{f} \in \mathbb{R}_{+}^{n}$, i.e.,

$$
\begin{aligned}
x_{f} & =\int_{0}^{t_{f}} \Phi\left(t_{f}-\tau\right) B \bar{u}(\tau) \mathrm{d} \tau \\
& =\int_{0}^{t_{f}} \Phi\left(t_{f}-\tau\right) B \hat{u}(\tau) \mathrm{d} \tau
\end{aligned}
$$

and

$$
\int_{0}^{t_{f}} \Phi\left(t_{f}-\tau\right) B[\bar{u}(\tau)-\hat{u}(\tau)] \mathrm{d} \tau=0
$$

By transposition of (16) and postmultiplication by $W^{-1}\left(t_{f}\right) x_{f}$, we obtain

$$
\int_{0}^{t_{f}}[\bar{u}(\tau)-\hat{u}(\tau)]^{T} B^{T} \Phi^{T}\left(t_{f}-\tau\right) \mathrm{d} \tau W^{-1}\left(t_{f}\right) x_{f}=0 .
$$

Substitution of (13) into (17) yields

$$
\begin{aligned}
\int_{0}^{t_{f}}[\bar{u}(\tau) & -\hat{u}(\tau)]^{T} B^{T} \Phi^{T}\left(t_{f}-\tau\right) \mathrm{d} \tau W^{-1} x_{f} \\
= & \int_{0}^{t_{f}}[\bar{u}(\tau)-\hat{u}(\tau)]^{T} Q \hat{u}(\tau) \mathrm{d} \tau=0 .
\end{aligned}
$$

Using (18), it is easy to verify that

$$
\begin{aligned}
\int_{0}^{t_{f}} & \bar{u}(\tau)^{T} Q \bar{u}(\tau) \mathrm{d} \tau \\
= & \int_{0}^{t_{f}} \hat{u}(\tau)^{T} Q \hat{u}(\tau) \mathrm{d} \tau \\
& \quad+\int_{0}^{t_{f}}[\bar{u}(\tau)-\hat{u}(\tau)]^{T} Q[\bar{u}(\tau)-\hat{u}(\tau)] \mathrm{d} \tau .
\end{aligned}
$$

From (19) it follows that $I(\hat{u})<I(\bar{u})$ since the second term on the right-hand side of the inequality is nonnegative. To find the minimal value of the performance index (11), we substitute (13) into (11) and obtain

$$
\begin{aligned}
I(\hat{u})= & \int_{0}^{t_{f}} \hat{u}^{T}(\tau) Q u(\hat{u}) \mathrm{d} \tau \\
= & x_{f}^{T} W^{-1} \int_{0}^{t_{f}} \Phi\left(t_{f}-\tau\right) B Q^{-1} \\
& B^{T} \Phi^{T}\left(t_{f}-\tau\right) \mathrm{d} \tau W^{-1} x_{f} \\
= & x_{f}^{T} W^{-1} x_{f}
\end{aligned}
$$

since (12) holds. 


\section{Procedure and a numerical example}

To find $t \in\left[0, t_{f}\right]$ for which $\hat{u}(t) \in \mathbb{R}_{+}^{n}$ reaches its minimal value using (13), we compute the derivative

$$
\frac{\mathrm{d} \hat{u}(t)}{\mathrm{d} t}=Q^{-1} B^{T} \Psi(t) W^{-1}\left(t_{f}\right) x_{f}, \quad t \in\left[0, t_{f}\right],
$$

where

$$
\Psi(t)=\frac{\mathrm{d}}{\mathrm{d} t}\left[\Phi^{T}\left(t_{f}-t\right)\right]
$$

Given $\Psi(t)$ and using the equality

$$
\Psi(t) W^{-1}\left(t_{f}\right) x_{f}=0,
$$

we can find $t \in\left[0, t_{f}\right]$ for which $\hat{u}(t)$ reaches its maximal value.

Note that if the system is asymptotically stable, $\lim _{t \rightarrow \infty} \Phi(t)=0$, then $\hat{u}(t)$ reaches its maximal value for $t=t_{f}$ and if it is unstable then for $t=0$.

From the above we have the following procedure for computation of optimal inputs satisfying the condition (10) that steer the state of the system from $x_{0}=0$ to $x_{f} \in \mathbb{R}_{+}^{n}$ and minimize the performance index (11).

\section{Procedure 1.}

Step 1. Given $A \in M_{n}$ and using (5), compute $\Phi(t)$.

Step 2. Using (12), compute the matrix $W\left(t_{f}\right)$ for given $A, B, Q, \alpha$ and some $t_{f}$.

Step 3. Using (13) and (23), find $t_{f}$ for which $\hat{u}(t)$ satisfying (10) reaches its maximal value and the desired $\hat{u}(t)$ for given $U \in \mathbb{R}_{+}^{n}$ and $x_{f} \in \mathbb{R}_{+}^{n}$.

Step 4. Using (15), compute the maximal value of the performance index.

Example 1. Consider the fractional positive system (2) for $\alpha=0.5$ with matrices

$$
A=\left[\begin{array}{ll}
2 & 0 \\
0 & 1
\end{array}\right], \quad B=\left[\begin{array}{ll}
0 & 1 \\
1 & 0
\end{array}\right],
$$

and the performance index (11) with

$$
Q=\left[\begin{array}{ll}
2 & 0 \\
0 & 2
\end{array}\right]
$$

Compute the bounded input $\hat{u}(t) \in \mathbb{R}_{+}^{2}$ satisfying the condition

$$
\hat{u}(t)=\left[\begin{array}{l}
\hat{u}_{1}(t) \\
\hat{u}_{2}(t)
\end{array}\right]<\left[\begin{array}{l}
1 \\
1
\end{array}\right] \quad \text { for } t \in\left[0, t_{f}\right]
$$

that steers the state of the system from zero state to $x_{f}=$ $\left[\begin{array}{ll}1 & 1\end{array}\right]^{T} \in \mathbb{R}_{+}^{2}$ ( $T$ denotes the transpose) and minimizes the performance index (11) with 25).

Using Procedure 1, we obtain what follows.
Step 1. From (5) and (24), we obtain

$$
\begin{aligned}
\Phi(t) & =\sum_{k=0}^{\infty} \frac{A^{k} t^{(k+1) \alpha-1}}{\Gamma[(k+1) \alpha]} \\
& =\sum_{k=0}^{\infty}\left[\begin{array}{cc}
2^{k} & 0 \\
0 & 1
\end{array}\right] \frac{t^{(k-1) 0.5}}{\Gamma[(k+1) 0.5]}
\end{aligned}
$$

Step 2. Using (12), (24) and (25), we get

$$
\begin{aligned}
W\left(t_{f}\right)= & \int_{0}^{t_{f}} \Phi\left(t_{f}-\tau\right) B Q^{-1} B^{T} \Phi^{T}\left(t_{f}-\tau\right) \mathrm{d} \tau \\
= & \int_{0}^{t_{f}} \Phi(\tau) B Q^{-1} B^{T} \Phi^{T}(\tau) \mathrm{d} \tau \\
= & \frac{1}{2} \int_{0}^{t_{f}} \Phi^{2}(\tau) \mathrm{d} \tau \\
= & \sum_{k=0}^{\infty}\left[\begin{array}{cc}
2^{k} & 0 \\
0 & 1
\end{array}\right] \frac{1}{\Gamma[(k+1) 0.5]} \int_{0}^{t_{f}} \tau^{k-1} \mathrm{~d} \tau \\
= & {\left[\begin{array}{cc}
1 & 0 \\
0 & 1
\end{array}\right] \frac{\ln t_{f}}{\Gamma(0.5)} } \\
& +\sum_{k=1}^{\infty}\left[\begin{array}{cc}
2^{2 k} & 0 \\
0 & 1
\end{array}\right] \frac{t_{f}^{k}}{k(\Gamma[(k+1) 0.5])^{2}} .
\end{aligned}
$$

Step 3. The system with (24) is unstable. Therefore, $\hat{u}(t)$ reaches its maximal value for $t=0$,

$$
\hat{u}(0)=Q^{-1} B^{T} \Phi^{T}\left(t_{f}\right) W^{-1}\left(t_{f}\right) x_{f}<\left[\begin{array}{l}
1 \\
1
\end{array}\right] .
$$

Using 29), we can find $t_{f}$ satisfying the condition 26,

$$
\begin{aligned}
\hat{u}(0)= & Q^{-1} B^{T} \Phi\left(t_{f}\right) W^{-1}\left(t_{f}\right) x_{f} \\
= & {\left[\begin{array}{cc}
0.5 & 0 \\
0 & 0.5
\end{array}\right]\left[\begin{array}{ll}
0 & 1 \\
1 & 0
\end{array}\right]^{T} } \\
& \times \sum_{k=0}^{\infty}\left[\begin{array}{cc}
2^{k} & 0 \\
0 & 1
\end{array}\right] \frac{t_{f}^{(k-1) 0.5}}{\Gamma[(k+1) 0.5]} \\
\times & {\left[\frac{\ln t_{f}}{\Gamma(0.5)}+\sum_{k=1}^{\infty} \frac{2^{k} t_{f}^{k}}{k(\Gamma[(k+1) 0.5])^{2}}\right.} \\
& \left.\times \frac{0}{\Gamma(0.5)}+\sum_{k=1}^{\infty} \frac{0}{k(\Gamma[(k+1) 0.5])^{2}}\right] \\
& {\left[\begin{array}{c}
1 \\
1
\end{array}\right] . }
\end{aligned}
$$




\section{Concluding remarks}

Necessary and sufficient conditions for the reachability of fractional positive continuous-time linear systems have been established (Theorem 2). The minimum energy control problem for fractional positive continuous-time linear systems with bounded inputs was formulated and solved. Sufficient conditions for the existence of a solution to the problem were given (Theorem 4) and a procedure for computation of an optimal input satisfying the condition (10) and the minimal value of the performance index was proposed. The effectiveness of the procedure was demonstrated on a numerical example. The presented method can be extended to positive discrete-time linear systems and to fractional positive discrete-time linear systems with bounded inputs.

\section{Acknowledgment}

This work was supported by a grant from the Białystok University of Technology (no. G/WE/1/11).

\section{References}

Busłowicz, M. (2008). Stability of linear continuous time fractional order systems with delays of the retarded type, Bulletin of the Polish Academy of Sciences: Technical Sciences 56(4): 319-324.

Dzieliński, A., Sierociuk, D. and Sarwas, G. (2009). Ultracapacitor parameters identification based on fractional order model, Proceedings of ECC'09, $\mathrm{Bu}$ dapest, Hungary.

Dzieliński, A. and Sierociuk, D. (2008). Stability of discrete fractional order state-space systems, Journal of Vibrations and Control 14(9/10): 1543-1556.

Farina, L. and Rinaldi, S. (2000). Positive Linear Systems: Theory and Applications, J. Wiley, New York, NY.

Kaczorek, T. (1992). Linear Control Systems, Research Studies Press and J. Wiley, New York, NY.

Kaczorek, T. (2001). Positive $1 D$ and $2 D$ Systems, Springer-Verlag, London.

Kaczorek, T. (2008a). Fractional positive continuous-time systems and their reachability, International Journal of Applied Mathematics and Computer Science 18(2): 223-228, DOI: 10.2478/v10006-008-0020-0.

Kaczorek, T. (2008b). Practical stability of positive fractional discrete-time linear systems, Bulletin of the Polish Academy of Sciences: Technical Sciences 56(4): 313-317.

Kaczorek, T. (2008c). Reachability and controllability to zero tests for standard and positive fractional discrete-time systems, Journal Européen des Systémes Automatisés 42(6-8): 769-787.

Kaczorek, T. (2009). Asymptotic stability of positive fractional 2D linear systems, Bulletin of the Polish Academy of Sciences: Technical Sciences 57(3): 289-292.
Kaczorek, T. (2011a). Controllability and observability of linear electrical circuits, Electrical Review 87(9a): 248-254.

Kaczorek, T. (2011b). Positivity and reachability of fractional electrical circuits, Acta Mechanica et Automatica 5(2): $42-51$.

Kaczorek, T. (2011c). Positive linear systems consisting of $n$ subsystems with different fractional orders, IEEE Transactions Circuits and Systems 58(6): 1203-1210.

Kaczorek, T. (2011d). Checking of the positivity of descriptor linear systems by the use of the shuffle algorithm, Archive of Control Sciences 21(3): 287-298.

Kaczorek, T. (2012). Selected Problems of Fractional Systems Theory, Springer-Verlag, Berlin.

Kaczorek, T. (2013a). Minimum energy control of fractional positive continuous-time linear systems, MMAR 2013, Międzyzdroje, Poland.

Kaczorek, T. (2013c). Minimum energy control of positive discrete-time linear systems with bounded inputs, Archives of Control Sciences 23(2): 205-211.

Kaczorek, T. (2013d). Minimum energy control of positive continuous-time linear systems with bounded inputs, International Journal of Applied Mathematics and Computer Science 23(4): 725-730, DOI: 10.2478/amcs-2013-0054.

Kaczorek, T. (2014a). Minimum energy control of descriptor positive discrete-time linear systems, COMPEL 33(3)

Kaczorek, T. (2014b). An extension of Klamka's method of minimum energy control to fractional positive discrete-time linear systems with bounded inputs, Bulletin of the Polish Academy of Sciences: Technical Sciences 62(2), (in press).

Kaczorek, T. and Klamka, J. (1986). Minimum energy control of 2D linear systems with variable coefficients, International Journal of Control 44(3): 645-650.

Klamka, J. (1976a). Relative controllability and minimum energy control of linear systems with distributed delays in control, IEEE Transactions on Automatic Control 21(4): 594-595.

Klamka, J. (1976b). Relative controllability and minimum energy control of linear systems with distributed delays in control, IEEE Transactions on Automatic Control 21(4): 594-595.

Klamka, J. (1977). Minimum energy control of discrete systems with delays in control, International Journal of Control 26(5): 737-744.

Klamka, J. (1983). Minimum energy control of 2D systems in Hilbert spaces, System Sciences 9(1-2): 33-42.

Klamka, J. (1991). Controllability of Dynamical Systems, Kluwer Academic Press, Dordrecht.

Klamka, J. (2010). Controllability and minimum energy control problem of fractional discrete-time systems, in D. Baleanu, Z.B. Guvenc and J.A. Tenreiro Machado (Eds.), New Trends Nanotechology and Fractional Calculus, Springer-Verlag, New York, NY, pp. 503-509.

Oldham, K.B. and Spanier, J. (1974). The Fractional Calculus, Academic Press, New York, NY. 
Ostalczyk, P. (2008). Epitome of the Fractional Calculus: Theory and Its Applications in Automatics, Technical University of Łódź Press, Łódź, (in Polish).

Podlubny, I. (1999). Fractional Differential Equations, Academic Press, San Diego, CA.

Radwan, A.G., Soliman, A.M., Elwakil, A.S. and Sedeek, A. (2009). On the stability of linear systems with fractional-order elements, Chaos, Solitons and Fractals 40(5): 2317-2328.

Solteiro Pires, E.J., Tenreiro Machado, J.A. and Moura Oliveira, P.B. (2006). Fractional dynamics in genetic algorithms, Workshop on Fractional Differentiation and Its Application, Porto, Portugal, Vol. 2, pp. 414-419.

Vinagre, B.M. (2002). Fractional order systems and fractional order control actions, IEEE CDC'02, Las Vegas, USA, NV, TW\#2, Lecture 3.

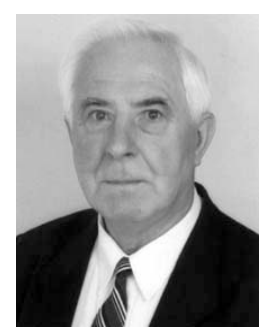

Tadeusz Kaczorek received the M.Sc., Ph.D. and D.Sc. degrees in electrical engineering from the Warsaw University of Technology in 1956, 1962 and 1964, respectively. In the years 1968-69 he was the dean of the Electrical Engineering Faculty, and in the period of 1970-73 he was a deputy rector of the Warsaw University of Technology. In 1971 he became a professor and in 1974 a full professor at the same university. Since 2003 he has been a professor at Białystok Technical University. In 1986 he was elected a corresponding member and in 1996 a full member of the Polish Academy of Sciences. In the years 1988-1991 he was the director of the Research Centre of the Polish Academy of Sciences in Rome. In 2004 he was elected an honorary member of the Hungarian Academy of Sciences. He has been granted honorary doctorates by ten universities. His research interests cover systems theory, especially singular multidimensional systems, positive multidimensional systems, singular positive 1D and 2D systems, as well as positive fractional $1 \mathrm{D}$ and $2 \mathrm{D}$ systems. He initiated research in the field of singular 2D, positive 2D and positive fractional linear systems. He has published 25 books (six in English) and over 1000 scientific papers. He has also supervised 69 Ph.D. theses. He is the editor-in-chief of the Bulletin of the Polish Academy of Sciences: Technical Sciences and a member of editorial boards of ten international journals.

Received: 24 August 2013

Revised: 29 November 2013 